\title{
HISTOPATHOLOGICAL CHANGES IN SOME REPRODUCTIVE AND ENDOCRINE ORGANS OF TRYPANOSOMA BRUCEI INFECTED WEST AFRICAN DWARF GOAT DOES
}

\author{
O. O. LEIGH ${ }^{1}$, B. O. EMIKPE ${ }^{2} \&$ J. O. OGUNSOLA ${ }^{2}$ \\ ${ }^{1}$ Department of Veterinary Surgery and Reproduction, ${ }^{2}$ Department of Veterinary \\ Pathology; Faculty of Veterinary Medicine, University of Ibadan, Nigeria
}

\begin{abstract}
Summary
Leigh, O. O., B. O. Emikpe \& J. O. Ogunsola, 2015. Histopathological changes in some reproductive and endocrine organs of Trypanosoma brucei infected West African dwarf goat does. Bulg. J. Vet. Med., 18, No 1, 31-39.

Available information on the mechanisms responsible for some reproductive lesions observed in experimental trypanosomiasis has not been fully elucidated. This work was undertaken to further study the histopathologic effects of Trypanosoma brucei infection on hypothalamus, adenohypophysis, ovary, uterus, placenta of the West African dwarf (WAD) doe and liver of aborted foetuses. Sixteen does (10 infected and 6 controls) and 3 foetuses from infected does were used for this study. Reproductive and endocrine organs from the does and livers of aborted foetuses were routinely processed for histopathology and studied using light microscopy. Findings revealed degenerative and inflammatory lesions which include hypothalamitis, adenohypophysitis, endometritis, placentitis as well as vacuolar change and cord atrophy in foetal liver. We conclude that experimental T. brucei infection caused significant histopathologies in the hypothalamus, adenohypophysis, uterus, placenta and foetal liver and that these lesions may underline the etiopathogenesis of infertility in trypanosome-infected WAD goat does.
\end{abstract}

Key words: endocrine, histopathology, reproduction, trypanosomosis, West African dwarf goat.

\section{INTRODUCTION}

Animal trypanosomiasis is endemic and responsible for an annual loss of $\$ 5$ billion revenue and threatens the lives of over 150 million cattle and small ruminants. It results in anaemia, decreased milk production as well as reduced reproductive abilities are consistent findings in ruminant trypanosomosis. These repro- ductive losses are often linked to unthriftiness, decreased rate of conception and pregnancy in affected cattle (Ogwu \& Nuru, 1981; Ogwu et al., 1986) and sheep (Reynolds \& Ekwuruke, 1988; Gabina, 1989). In female animals, severe genital lesions (Ikede et al., 1988) which results in abnormal oestrous cycles, death during 
Histopathological changes in some reproductive and endocrine organs of Trypanosoma brucei....

pregnancy, dystocia, abortion, premature birth, low birth weight, stillbirth, transplacental infection, neonatal death and other pathogenic effects on foetuses had been reported (Sekoni, 1994; Bawa et al., 2000; Faye et al., 2004). Abundant literature exists on the pathogenesis of the anaemia while the exact mechanisms resulting in persistent reproductive losses have not been thoroughly elucidated. There had been a report of the association of trypanosomiasis with sperm and zona pellucida antibodies (Fayemi, 2003). Other researchers have earlier identified the pathogenesis of the reproductive failures in trypanosomosis to include pituitary hormonal defects or pituitary dysfunction (Apted, 1970; Isoun \& Anosa, 1974; Griffin \& Allonby, 1979) while some other researchers reported the absence of gross and histologic lesions in the endocrine and reproductive organs of infected animals (El-Hassan et al., 1994). However, Reincke et al. (1998) observed polyglandular endocrine failure by local inflammation of pituitary and gonadal glands in experimental trypanosome infection. Extensive hyalinisation of ovarian stroma, extravasation of blood in the hypothalamus and mononuclear cellular infiltration into the capsule and parenchyma of the hypophysis were also reported in ewes infected with Trypanosoma vivax (Adenowo et al., 2005). Other proposed mechanism includes oxidative stress resulting from excessive generation of free radicals and super-oxides (Igbokwe, 1994). Available literature on lesions observed in experimental infections with trypanosomes has not fully explained the mechanism responsible for some of the reported reproductive conditions. Although these lesions may not be significant enough, they may underline the basis of and provide some insight into the reproductive pathology earlier reported.

To our knowledge, the histopathology of key endocrine organs, uterus of the West African dwarf (WAD) doe and livers of aborted fetuses have not been studied. This work was undertaken to further study the effects of Trypanosoma brucei experimental infection on hypothalamus, adenohypophysis, ovary, uterus, placenta and foetal liver in WAD does.

\section{MATERIALS AND METHODS}

\section{Experimental animals}

Sixteen (16) adult WAD does weighing between 16.0 and $17.5 \mathrm{~kg}$ were purpsefully selected for the trial. The goats were initially screened and found negative for trypanosomes via HCT and Buffy coat method (Woo, 1971). The does were introduced to the pen of the bucks for about 48 hrs starting from $72 \mathrm{~h}$ post synchronisation with Lutalyse for natural mating to occur. Pregnancy was diagnosed: via trans-abdominal ultrasonography using real-time B-mode scanner with 3.5$5 \mathrm{MHz}$ transducer. The control (6) and infected (10) groups of does were made up of equal halves $(50 \%)$ of gravid and non-gravid does.

\section{Parasite and infection}

The parasite, Kaura strain of Trypanosoma brucei brucei, was obtained from the Nigerian Institute for Trypanosomiasis Research (NITR), Vom, Jos. The parasite was maintained in rats, not included in the present experiments, by repeated passaging. Parasitaemia was monitored and detected by placing a drop of blood from the tail vein of the infected rats, coverslipped and viewed under $\times 100$ magnification. A semi-quantitative chart adapted 
for use in the Clinical Pathology laboratory of the Department of Veterinary Pathology, was used to assess the level of parasitaemia in the rats.

Parasite-infected blood was obtained from the tail of infected rats with high parasitaemia levels and used to maintain parasite suspension in $0.9 \%$ normal saline solution. The suspension contained about 2-3 trypanosomes per field when viewed under $\times 100$ magnification. Of this $T$. brucei suspension, $0.5 \mathrm{~mL}$ was injected in the jugular vein of the goats through the $2^{\text {nd }}$ trimester (i.e. day 51 post-mating) and the duration of infection was 32-46 days (Leigh \& Fayemi, 2013).

Monitoring of development and maintenance of parasitaemia in the infected WAD does was done using a wet mount of drop of blood obtained from the jugular vein at day 5 and 9 post-inoculation. The wet mount was cover-slipped and viewed at $\times 100$. The degree of parasitaemia was determined using the semiquantitative chart adapted for use at the lab.

\section{Histopathologic examination}

Control and infected animals were euthanised and necropsy was performed on the sixteen WAD does. The hypothalamus, adenohypophysis, ovaries, uterus and placenta were initially collected in Bouin's fluid for 24 hours before they were transferred into $10 \%$ formalin for fixation. In relevant cases, the whole foetus was also collected and the foetal liver was removed by dissection and fixed as described above. The tissues were processed for histology and stained with H\&E (Junqueira \& Carneiro, 1980). Sections (5 $\mu \mathrm{m})$ were examined with an Olympus ${ }^{\circledR}$ microscope. Photomicrographs were obtained with the aid of a digital camera fitted to the microscope while scale bars were inserted with the ImageJ software.

\section{RESULTS}

Lesions were observed in the hypothalamus, pituitary, uterus and foetal liver while there was no visible lesion in the ovary. The detailed histopathological findings for the organs are presented below.

\section{Hypothalamus}

In the infected does, there was marked widespread congestion of blood vessels. In addition, there were severe perivascular lymphocytic cuffs (Fig. 1A). A few neurons showed moderate neuronal body swelling and degeneration.

\section{Pituitary gland}

The pars distalis in the uninfected controls was unremarkable while the pars intermedia contained numerous well-formed colloid-containing cavities (Fig. 1B). However, in the infected does, there was a subacute necrotising adenohypophysitis characterised by widespread necrosis and disruption of the architecture of the adenohypophysis. There were multiple foci of inflammatory cells which were mostly lymphocytes and a few neutrophils (Fig. 1C). There was marked vascular congestion and severe lymphocytic perivascular cuffs. Mild fibroplasia and no colloidcontaining cavities were also observed.

\section{Ovaries}

The ovaries in the uninfected controls as well as the infected does showed no remarkable lesions.

\section{Uterus}

In $T$. brucei infected does, there was a necrotising acute severe endometritis characterised by marked extensive necro- 

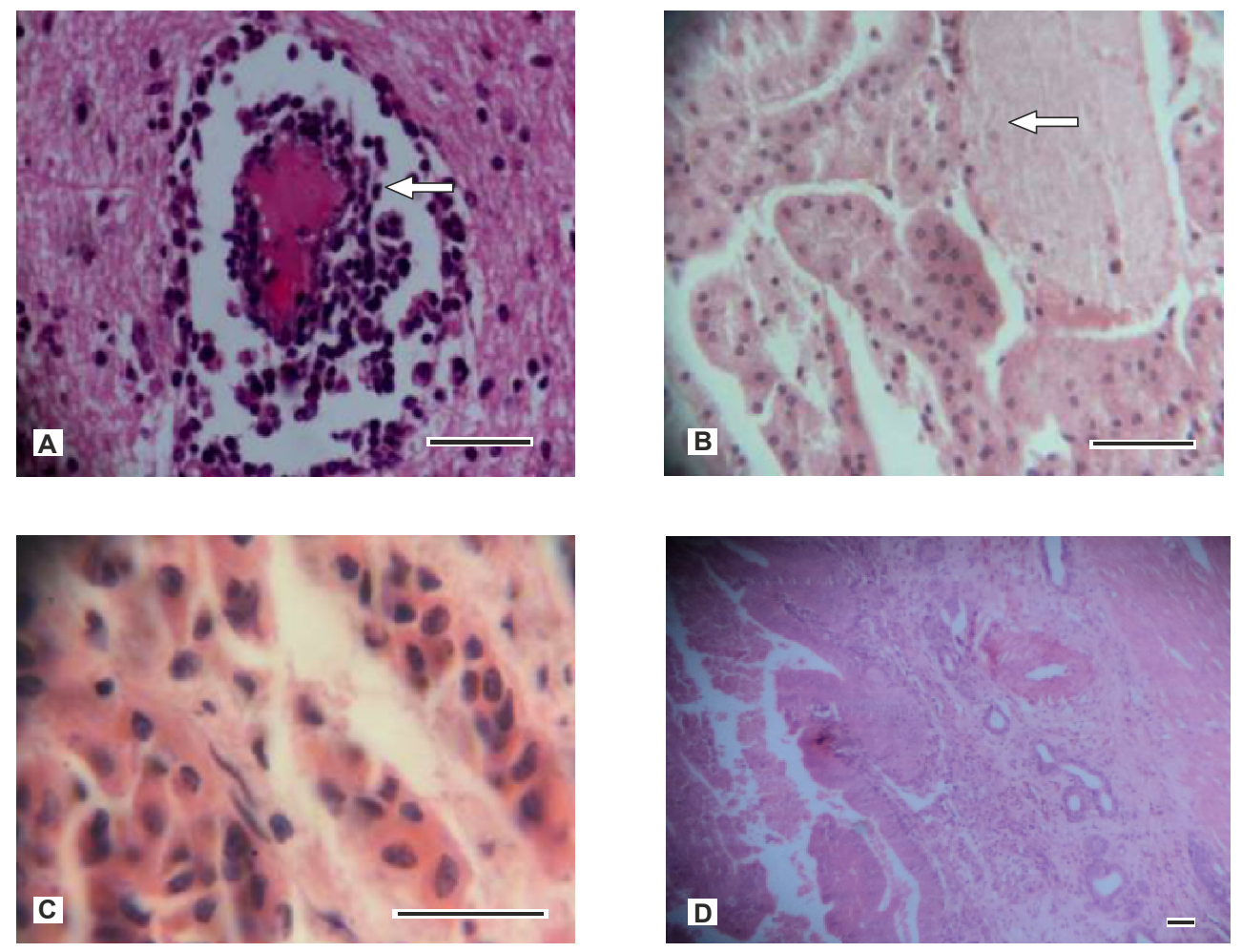

Fig. 1. A. Hypothalamus. Marked vascular congestion and severe lymphocytic perivascular cuffs in a doe infected with T. brucei $(\mathrm{H} \& \mathrm{E}$, bar $=100 \mu \mathrm{m})$. B. Adenohypophysis. Pars intermedia containing numerous colloid-filled cavities in a control doe $(\mathrm{H} \& \mathrm{E}, \mathrm{bar}=100 \mu \mathrm{m})$. C. Adenohypophysis. Severe widespread disruption of architecture with moderate aggregates of inflammatory cells (lymphocytes, neutrophils and a few fibroblasts) in a doe infected with T. brucei (H\&E, bar=100 $\mu \mathrm{m})$. D. Uterine horn. Extensive necrosis of epithelium and endometrial glands. Lamina propria contains numerous inflammatory lymphocytes, in an infected doe. Note that the myometrium is unaffected (H\&E, bar $=100 \mu \mathrm{m})$.

sis of the epithelium with varying degrees of necrosis and distortion of the tubular glands in the endometrial stroma (Fig. 1D). The blood vessels of the endometrial stroma were markedly congested while the lamina propria was oedematous and contained numerous foci of marked inflammatory cellular aggregates comprising of mostly lymphocytes and neutrophils and to a lesser extent, a few macrophages and plasma cells (Fig. 2A). No lesions were observed in the myometrium and serosa of the uterus. The uterus was devoid of lesions in uninfected does.

\section{Placenta}

An acute placentitis characterised by marked vascular congestion and multifocal lymphocytic aggregates was observed in $T$. brucei infected pregnant does (Fig. 2B).

\section{Foetal liver}

There was marked widespread thinning of the hepatic plates (cord atrophy) and diffuse moderate vacuolar change of existing 

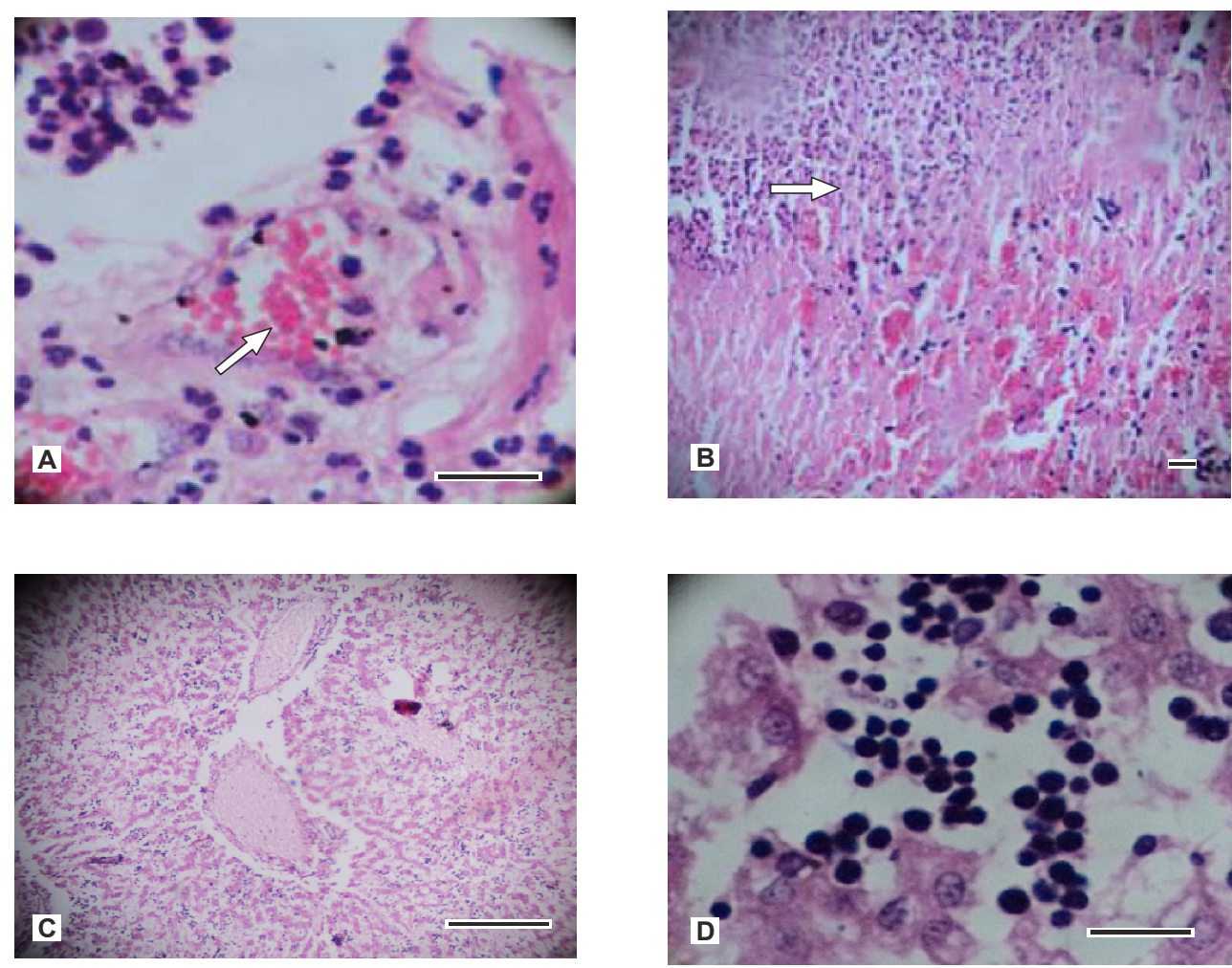

Fig. 2. A. Endometrial stroma: There are numerous inflammatory cells which are mostly lymphocytes, macrophages, neutrophils and a few occasional plasma cells. Note the congestion of blood vessels $(\mathrm{H} \& \mathrm{E}$, bar $=100 \mu \mathrm{m})$. B. Placenta: Acute placentitis characterised by moderate congestion of blood vessels (thin arrow) and numerous foci of inflammatory aggregates (mostly lymphocytes) (large arrow) in an infected doe (H\&E, bar=100 $\mu \mathrm{m})$. C. Foetal liver. Marked widespread thinning of hepatic cords and moderate vacuolar change of hepatocytes (H\&E, bar $=100$ $\mu \mathrm{m})$. D. Foetal liver. Haemopoietic focus. Pyknosis and karyorrhexis of the nuclei of the haemopoietic cells. Note the absence of a graded maturation sequence $(\mathrm{H} \& \mathrm{E}$. bar $=100 \mu \mathrm{m})$.

hepatocytes (Fig. 2C). The foci of haemopoiesis in livers of foetuses of infected does revealed numerous cells with pyknotic and karyorrhectic nuclei (Fig. 2D).

\section{DISCUSSION}

The lesions associated with experimental T. brucei infection in this study included hypothalamitis, adenohypophysitis, endometritis and placentitis in the does as well as hepatocellular degeneration, cord atrophy and dyshaemopoiesis in the foetuses. Although T. brucei is both haematic and tissue-invading trypanosome, parasites were not found in the tissues examined but they elicited inflammatory responses in these tissues. The mechanism by which trypanosomes evade the blood brain barrier to spread to the pituitary gland is not clear (Chirimwani et al. 1988). The observed inflammatory responses of the 
hypothalamus and adenohypophysis were much similar to histological changes described in similar organs in $T$. vivaxinfected sheep by Adenowo et al. (2005).

The lesions observed are capable of leading to aberrations in the production of gonadotropin-releasing hormone $(\mathrm{GnRH})$, insufficient to stimulate the production of gonadotrophins, or may cause direct reduction in the functional potential of the adenohypophysis which could result in absence of stimulation of ovarian cycles since initiations of ovarian cycles depend on the availability of sufficient pituitary gonadotrophins (Ball \& Peters, 2004). Though hormonal changes were not evaluated in this study, Leigh \& Fayemi (2013) had reported reduction in the concentrations of follicle stimulating hormone, during oestrus, and luitenizing hormone, during proestrus and oestrus, as well as oestradiol, during the follicular phase in $T$. brucei-infected WAD does. These findings according to the study were suggested to be contributory to the observed anoestrus in infected does. It is however not clear if the reduction in the concentration of oestradiol observed in that study has any relationship with degeneration of ovarian follicles reported by Rodriguez et al. (2013) in T. vivaxinfected goats; but Reincke et al. (1998) had reported polyglandular endocrine failure by local inflammation of the pituitary and gonadal glands in trypanosomosis. Although, trypanosomes were not clearly demonstrated in the adenohypophysis, inflammation and disruption of tissue architecture as observed in this study could have been precipitated by direct presence of $T$. brucei as reported by Damayanti (1998).

The reason why there were no remarkable lesions in the ovary may be that the blood-ovary barrier had not been breached. The lesion observed in the uterine body, a severe necrotising endometritis, is capable of reducing its potential to carry out its physiological functions which include the production of PGF $2 \alpha$ (i.e. luteolysin) and nidation. The result of the earlier would be anoestrus as a result of continuous production of progesterone from an active corpus luteum while that of the latter could be reduced ability of the uterus to support the growth of any foetus(es). An indication suggestive of failure in these physiological functions of the endometrium could be inferred from our ealier study (Leigh \& Fayemi, 2013) where T. brucei infection led to sustained corpus luteum activity in synchronised does and the spate of abortion observed. Also, the necrosis of endometrial glands would result in the absence of endometrial milk that is necessary for the nourishment of the implanted foetus. Lesions observed in the placenta in the study corroborate those reported by Silva et al. (2013) in $T$. vivax-infected sheep. These lesions are capable of interfering with normal exchanges between foetus and dam, leading to foetal hypoxia and death in utero or the initiation of early dehiscence of the placenta and subsequent abortion (Dubey et al., 2006). Rocha et al. (2004) asserted that $T$. brucei possesses the ability to traverse the placenta causing a placentitis and also infection in utero. The placentitis observed in the doe may well explain cord atrophy due to impaired nutritional and gaseous exchanges between the dam and the foetus as opined by Marcato et al., (1991).

The marked vacuolar change of foetal hepatocytes may be due to anoxia, which may have arisen partly due to placentitisinduced impaired gaseous exchange or partly due to the persistent anaemia in the 
dam that characterise trypanosomal infection.

The possibility that both mechanisms contribute to the foetal anoxia cannot be completely ruled out. Dyshaemopoiesis or ineffective haemopoiesis found in the foetal liver may just mirror what happens in the adult bone marrow in trypanosomosis. Strictly speaking, ineffective erythropoiesis resulting from necrosis of erythroid precursors or inadequate response of these precursors to erythropoietin has been implicated as a cause of the anaemia that has consistently characterised trypanosomosis. Across all the organs studied (except the ovaries), we noticed two persistent features. First, T. brucei induced a necrotising inflammatory response with varying degrees of severity. This presumes the presence and/or eliciting of a necrotising factor(s). Further studies would be required to determine if this necrotising factor is host-derived or parasite-derived. Second, is the nature of the inflammatory cellular response. In all organs, the lymphocyte was the predominant cell type. This finding suggests that $T$. brucei infection stimulates a strongly immunologic response and this is not surprising as workers are familiar with the parasitaemic waves that coincide with the expression of a different variable surface glycoprotein (VSG) by the trypanosome. This ultimately leads to immunologic exhaustion.

We conclude that $T$. brucei experimental infection caused significant histopathological alterations in the hypothalamus, adenohypophysis, uterus, placenta and foetal liver, suggestive of the involvement of the parasite in the etiopathogenesis of infertility in WAD goat does.

\section{ACKNOWLEDGEMENTS}

The authors sincerely acknowledge the support and guidance of Prof. O. Eric Fayemi, now of Michael Okpara University, Umudike, Nigeria.

\section{REFERENCES}

Adenowo, T. K., C. O. Njoku, E. O. Oyedipe \& A. Sannusi, 2005. Lesion of the hypothalamus, adenohypophysis and the ovaries in Trypanosoma vivax infected Yankasa Ewes. Nigerian Veterinary Journal, 26, 56-62.

Apted, F. I. C., 1970. Clinical manifestations and diagnoses of sleeping sickness. In: The African Trypanosomiasis, ed. H. W. Mulligan, George Allen and Unwin, London. pp. 667.

Ball, P. J. H. \& A. R. Peters, 2004. Reproduction in Cattle. Blackwell Publishing Company, USA. pp. 427.

Bawa, E. K., D. Ogwu, V. O. Sekoni, E. O. Oyedipe, K. A. Esievo \& J. E. Kambai, 2000. Effects of Trypanosoma vivax on pregnancy of Yankasa sheep and the results of homidium chloride chemotherapy. Theriogenology, 54, 1033-1040.

Chirimwani, B., E. A. E. Van Marck, J. M. Brucher, M. Wery \& P. L. J. Gigase, 1988. Progression of central nervous system lesions in the rat infected with Trypanosoma brucei-gambiense: A light microscopic study. Annales de la Société Belge de Médecine Tropicale, 68, 205-218.

Damayanti, R., 1998. Identification of Trypanosoma evansi in infected rat tissues by immunohistochemical methods. Penyakit Hewan, 25, 11-13.

Dubey, J. P., D. Buxton \& W. Wouda, 2006. Pathogenesis of bovine neosporosis. Journal of Comparative Pathology, 134, 267289.

El-Hassan, E., B. O. Ikede \& O. Adeyemo, 1994. Trypanosomiasis and reproduction: 1. Effect of Trypanosoma vivax infection on the oestrous and fertility in the ewe. 
Histopathological changes in some reproductive and endocrine organs of Trypanosoma brucei....

Tropical Animal Health and Production, 26, 213-218.

Faye, D., J. Sulon, Y. Kane, J. F. Beckers, S. Leak, Y. Kaboret, N. M. de Sousa, B. Losson \& S. Geerts, 2004. Effects of an experimental Trypanosoma congolense infection on the reproductive performance of West African Dwarf goats. Theriogeno$\log y$, 62, 1438-1451.

Fayemi, O., 2003. Association of Trypanosome infection with zona pellucida antibodies in goats. African Journal of Biomedical Research, 6, 137-140.

Gabina, D., 1989. Improvement of the reproductive performance of Rasa Aragonesa flocks in frequent lambing systems. I. Effects of management system, age of ewe and season. Livestock Production Science, 22, 69-85.

Griffin, L. \& E. W. Allonby, 1979. Studies on the epidemiology of trypanosomiasis in sheep and goats in Kenya. Tropical Animal Health and Production, 11, 133-142.

Igbokwe, I. O., 1994. Mechanism of cellular injury in African trypanosomiasis. Veterinary Bulletin, 64, 611-615.

Ikede, B. O., E. El-Hassan \& S. O. Akpavie, 1988. Reproductive disorders in African trypanosomasis: A review. Acta Tropica, 45, 5-10.

Isoun, T. T. \& V. O. Anosa, 1974. Lesions in the reproductive organs of sheep and goats infected with $T$. vivax. Tropenmedizin und Parasitologie, 25, 469-476.

Junqueira, L. C. \& J. Carneiro, 1980. Basic Histology, $3^{\text {rd }}$ edn, Lange Medical Publications California, pp. 1-14.

Leigh, O. O. \& O. E. Fayemi, 2013. The effects of experimental Trypanosoma brucei infection on hormonal changes during the oestrous cycle, pregnancy and pregnancy outcome in West African dwarf goat does. Wayamba Journal of Animal Science, 5, 685-695.

Marcato, P. S., C. Benazzi, G. Vecchi, M. Galeotti, D. L. Salda, G. Sarli, \& P. Lucidi, 1991. Clinical and pathological features of viral haemorrhagic disease of rabbits and the European brown hare syndrome. Revue Scientifique et Technique de l'Office International des Epizooties, 10, 371-392.

Ogwu, D. \& S. Nuru, 1981. Transplacental transmission of trypanosomes in animals and man. A Review. The Veterinary Bulletin, 51, 381-384.

Ogwu, D., C. O. Njoku \& D. I. K. Osori, 1986. Effects of experimental $T$. vivax infection during second and third trimester pregnancy in heifers. Theriogenology, 34, 383398.

Reincke, M., W. Arlt, C. Heppner, F. Petzke, G. P. Chrousos, \& B. Allolio, 1998. Neuroendocrine dysfunction in African trypanosomiasis: The role of cytokines. Annals of the New York Academy of Science, 840, 809-821.

Rocha, G., A. Martins, G. Gama, F. Brandao. \& J. Atouguia, 2004. Possible cases of sexual and congenital transmission of sleeping sickness. The Lancet, 363, 247.

Reynolds, L. \& J. O. Ekwuruke, 1988. Effects of Trypanosoma vivax infection on West African dwarf sheep at two planes of nutrition. Small Ruminant Research, 1, 175-188.

Rodriguez, C. M. F., R. G. Olinda, T. M. F. Silva, R. G. Vale, A. E. Silva, G. L. Lima, H. A. Garcia, M. Teixeira, \& J. S. Batista, 2013. Follicular degeneration in the ovaries of goats experimentally infected with Trypanosoma vivax from the Brazilian semi-arid region. Veterinary Parasitology, 191, 146-153.

Sekoni, V. O., 1994. Reproductive disorders caused by animal trypanosomiasis. A review. Theriogenology, 42, 557-570.

Silva, T. M. F., R. G. Olinda, C. M. F. Rodrigues, A. C. L. Camara, F. C. Lopes, W. A. C. Coelho, M. F. B. Ribeiro, C. I. A. Freitas, M. M. G. Teixeira \& J. S. Batista, 2013. Pathogenesis of reproductive failure induced by Trypanosoma vivax in experimentally infected pregnant ewes. Veterinary Research, 44, 1. 
Woo, P. T. K., 1971. Evaluation of haematocrit centrifuge and other techniques for field diagnosis of trypanosomiasis and filariasis. Acta Tropica, 28, 298-303.

Paper received 13.05.2014; accepted for publication 13.06.2014

\section{Correspondence:}

Dr. O. O. Leigh

Department of Veterinary Surgery and

Reproduction,

Faculty of Veterinary Medicine,

University of Ibadan, Nigeria.

tel: +2348033266825

e-mail: damilareolufisayo@yahoo.com;

damilareolufisayoleigh@gmail.com 